\title{
Modified Cross Feedback Control for a Magnetically Suspended Flywheel Rotor with Significant Gyroscopic Effects
}

\author{
Yuan $\operatorname{Ren}^{1,2}$ and Jiancheng Fang ${ }^{2}$ \\ ${ }^{1}$ Department of Space Equipment, Equipment Academy, Beijing 101416, China \\ ${ }^{2}$ School of Instrumentation Science \& Opto-Electronics Engineering, Beihang University, Beijing 100191, China \\ Correspondence should be addressed to Yuan Ren; renyuan_823@aliyun.com
}

Received 25 July 2013; Revised 12 November 2013; Accepted 26 November 2013; Published 14 January 2014

Academic Editor: Xinkai Chen

Copyright (c) 2014 Y. Ren and J. Fang. This is an open access article distributed under the Creative Commons Attribution License, which permits unrestricted use, distribution, and reproduction in any medium, provided the original work is properly cited.

\begin{abstract}
For magnetically suspended rigid rotors (MSRs) with significant gyroscopic effects, phase lag of the control channel is the main factor influencing the system nutation stability and decoupling performance. At first, this paper proves that the phase lag of the cross channel instead of the decentralized channel is often the main factor influencing the system nutation stability at high speeds. Then a modified cross feedback control strategy based on the phase compensation of cross channel is proposed to improve the stability and decoupling performances. The common issues associated with the traditional control methods have been successfully resolved by this method. Analysis, simulation, and experimental results are presented to demonstrate the feasibility and superiority of the proposed control method.
\end{abstract}

\section{Introduction}

High speed flywheel systems are being widely employed in power industry, aerospace, transportation, military applications, and so on, which show promise as an alternative to energy storage and attitude control for spacecraft, such as storage energy flywheels, reaction wheels, and momentum flywheels in control moment gyroscopes [1-3]. With the advance of high performance magnetic bearings, a magnetically suspended flywheel (MSFW) is becoming an exciting alternative to the traditional mechanical flywheel due to its inherent superior features such as contact-free operation, small noise, and adjustable damping and stiffness as well as the potential for low vibration and super high rotational speeds [4-7].

To maximize the energy storage capacity, the magnetically suspended rotor (MSR) is often designed as a plot structure [8]. In this case, the gyroscopic effects are especially significant, which puts a challenging issue on the highstability and high-precision control of the system. Recently the demand for higher power density and efficiency has led to more significant gyroscopic effects and higher operating speed, making the stability control more difficult $[9,10]$.
Over the years, considerable research has been conducted to resolve the gyroscopic effects, and various approaches have been proposed. Ahrens et al. [11] proposed decentralized PID plus speed cross feedback control approach to attain this goal. Although this method is relatively simple to implement, it inevitably introduces noise amplification due to derivative operation. Brown et al. [12] further presented the filtered cross-axis proportional gains method, which not only can greatly improve the stability of the gyroscopic modes, but also can effectively avoid excessive noise enhancement caused by the cross feedback arithmetic. Unfortunately, this method is relatively complex due to multicoordinate transformation. In addition, to realize the separate control of the stiffness and damping of the rotor's rigid modes, Dever et al. [13] proposed a modal controller. However, this method cannot realize the decoupling between the rotation modes of the MSR. Accordingly, it cannot reject radically the gyroscopic effects. To realize the high-precision control of a magnetically suspended control moment gyro (MSCMG), Fang and Ren [6] proposed a channel decoupling strategy based on current-mode inverse system method and phase compensation filters. This method can realize the decoupling control among the four radial channels of the MSR. Nevertheless, 
this decoupling algorithm is not away from complexity because of complex inversion arithmetic and the combined robust controllers. To overcome the shortcomings of the channel decoupling method, the modal decoupling control based on modified modal separation and rotation motions decoupling strategy has been further proposed [17]. Although the robustness and simplicity have been improved greatly, it is relatively complex compared with the traditional cross feedback control.

All in all, the common issues of the existing methods mainly lie in the contradictions among the high decoupling precision, high stability, and simplicity.

Moreover, all the methods above have some difficulty in achieving satisfactory control performances at high rotor speeds due to phase lag of the control system, which even endangers the whirling mode stability [14]. There are three main factors which introduce phase lag into the control system. The first one is all kinds of low-pass filters, such as digital or analog antialias filters employed to cut off the frequency components above the band of interest. The second one is the switching power amplifiers delay since the magnetic bearings are typical inductive loads. The third one is the digital control delay. Among these, the switching power amplifier delay is the dominating factor as for a high speed MSR system.

Therefore, to improve the system nutation stability and control performances, it is necessary to develop phase compensation for the control system. In early work $[6,8,9]$, highpass filters to compensate for the unmodeled dynamic of the system have been employed to improve the decoupling performance of the current-mode inverse system method. However, this method inevitably causes large computation resources and noise amplification. Ren and Fang [15] proposed a phase compensation method based on unsymmetrical sampling resistance networks to decrease the system phase lag resulted by the coil inductance and digital control delay for a magnetic bearing switching power amplifier. Although this method does not induce extra computation resources, it also results in noise amplification since all the coupling channels need to perform phase compensation. Concretely speaking, when these methods are employed in a 5-degreeof-freedom (DOF) MSR, they need to compensate for the four radial channels, that is, four coupling channels. For the sake of the convenience, the phase compensation methods mentioned above are collectively called the coupling channel compensation method in this paper.

In this paper, the solution is oriented to resolve the common issues above. Based on the decentralized PID plus cross feedback control, a modified cross feedback control strategy is proposed to improve the system nutation stability and decoupling precision without extra computation effort and system noise. The main contribution of this paper lies in that the common contradictions of the existing control methods for a MSR can be successfully resolved with the proposed method.

The remainder of this paper is organized as follows. First, in Section 2, the system is modeled and its phase lag characteristics are analyzed. Then, the proof is developed and the modified cross feedback control strategy based

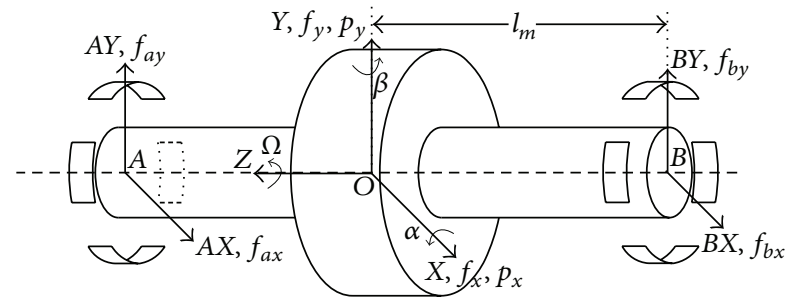

FIGURE 1: Rotor forces and the coordinate systems.

on phase compensation of cross channel is proposed in Section 3. Comparative simulation and experiments between the modified method and the traditional one (coupling channel compensation method) are developed in Section 4. Finally, Section 5 concludes this paper.

\section{Modeling of the Whole Control System for MSR}

2.1. Dynamic Modeling of the Cross Feedback Controller. Here we review the model of a MSR, which has been well established [6]. A schematic of the rotor forces and the coordinate systems is shown in Figure 1, where $O$ is the geometric center of the magnetic bearing stator; $x_{-}, y_{-}$, and $z$-axes form the generalized coordinate system of the rotor position; $\alpha$ and $\beta$ are the rotor angular displacements about the $x$ - and $y$-axes. $f_{a x}, f_{a y}, f_{b x}$, and $f_{b y}$ are the magnetic forces along the magnetic bearing coordinate system $A X-, A Y-, B X-$, and $B Y$-axes; $f_{x}$ and $f_{y}$ are the magnetic forces in the $x$-and $y$-axes, respectively; $p_{x}$ and $p_{y}$ are the torques in the $x$ - and $y$ directions; $\Omega$ is the rotor speed of the MSR; $l_{m}$ is the distance from $O$ to the center of the radial magnetic bearing.

According to Newton's second law and the principle of rotor dynamics, the dynamic model of the rigid rotor supported by magnetic bearings can be described as

$$
\begin{gathered}
m \ddot{x}=f_{a x}+f_{b x}, \\
J_{y} \ddot{\beta}-J_{z} \Omega \dot{\alpha}=p_{y}=l_{m}\left(f_{a x}-f_{b x}\right), \\
m \ddot{y}=f_{a y}+f_{b y}, \\
J_{x} \ddot{\alpha}+J_{z} \Omega \dot{\beta}=p_{x}=l_{m}\left(f_{b y}-f_{a y}\right),
\end{gathered}
$$

where $J_{x}, J_{y}$ and $J_{z}$ are the moments of inertia of the rotor about the $x$-, $y$-, and $z$-axes, respectively; $x$ and $y$ are the linear displacements of the mass center of the rotor from $O$ in the $x$ - and $y$-axes.

Figure 2 shows the schematic of the cross feedback controller, where $g_{d}(s)$ and $g_{c r}(s)$ are the transfer functions of the basic controller (such as a PID controller) and the cross feedback controller; $g_{f}(s)$ is the transfer function of the filters, including the antialias filter and notch filter; $g_{\tau}(s)$ is the delay unit of the control channel; $g_{\tau}(s)=e^{-\tau s}, k_{c}$ and $k_{h}$ denote, respectively, the force-current factor and forcedisplacement factor of the radial magnetic bearings; $k_{s}$ is the proportional coefficient of the current sensor. 


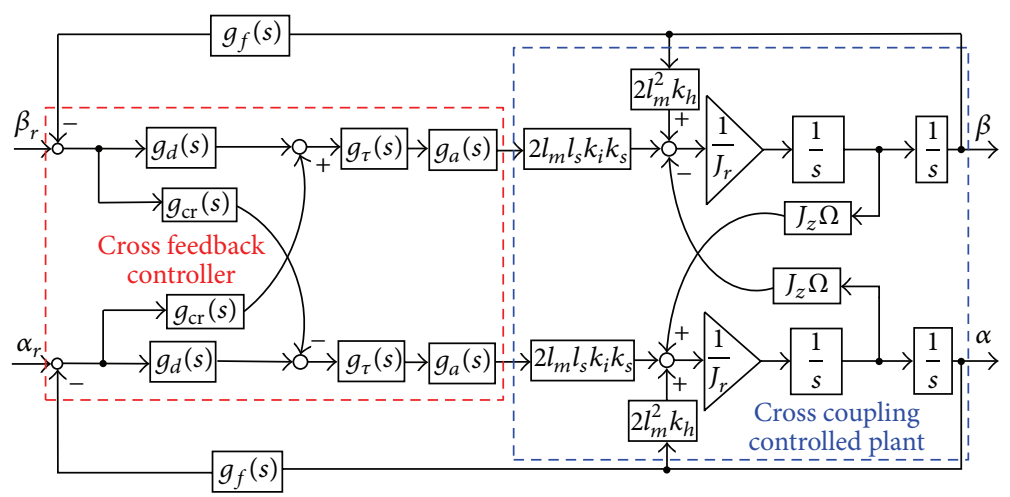

Figure 2: Schematic diagram of decentralized control plus cross feedback.

Combing the dynamic equation of the MSR, the rotational modes of the closed-loop MSR system based on cross feedback controller can be described as follows:

$$
\begin{aligned}
J_{y} \ddot{\beta}(t) & -H \dot{\alpha}(t)-2 k_{h} l_{m}^{2} \beta(t) \\
= & -2 l_{m} l_{s} k_{c} k_{s} g_{a} g_{\tau} g_{f}\left[g_{d} \beta(t)+2 g_{\mathrm{cr}} \alpha(t)\right], \\
J_{x} \ddot{\alpha}(t) & +H \dot{\beta}(t)-2 k_{h} l_{m}^{2} \alpha(t) \\
= & -2 l_{m} l_{s} k_{c} k_{s} g_{a} g_{\tau} g_{f}\left[g_{d} \alpha(t)-2 g_{\mathrm{cr}} \beta(t)\right],
\end{aligned}
$$

where $g_{a}(s)$ and $g_{f}(s)$ denote the power amplifier and the antialias filter, respectively, $l_{s}$ is the distance from $O$ to the center of the radial displacement sensor, and $H=J_{z} \Omega$.

\subsection{Characteristic Analysis of Phase Lag of the Control System.} According to the coordinate system definition shown in Figure 1, the rotating direction of the rotor speed is from $x$-axis to $y$-axis. Note that $\alpha$ and $\beta$ are the rotor angular displacements about the $x$ - and $y$-axes, respectively, and $X$ axis and $Y$-axis are upright. Accordingly, $\alpha$ leads $\beta$ by 90 degrees. Define $\varphi=\alpha+j \beta$, where $j$ is the imaginary number unit and $j^{2}=-1$. As for the symmetrical magnetic bearing, $J_{x}=J_{y}$. Note that the antisymmetry of (2), multiplying the first equation by $j$ and then adding the result to the second one yields,

$$
\begin{aligned}
J_{r} \ddot{\varphi}(t) & -j H \dot{\varphi}(t)-2 k_{h} l_{m}^{2} \varphi(t) \\
= & -2 l_{m} l_{s} k_{c} g_{a} g_{\tau} g_{f}\left(g_{d}+j 2 g_{\mathrm{cr}}\right) \varphi(t),
\end{aligned}
$$

where $J_{r}=J_{x}=J_{y}$.

The above equation is the differential equation of the rotation modes with the decentralized control plus cross feedback. Its Laplace conversion can be resolved as

$$
\begin{aligned}
J_{r} s^{2}- & j H s-2 k_{h} l_{m}^{2} \\
& =-2 l_{m} l_{s} k_{c} k_{s} g_{a}(s) g_{f}(s)\left(g_{d}(s)+j 2 g_{\mathrm{cr}}(s)\right) e^{-\tau s} .
\end{aligned}
$$

Then the original systems described as (4) can be equivalent to a feedback control system. Its equivalent controlled plant and equivalent transfer function of the control channel can be described, respectively, as

$$
\begin{gathered}
H(s)=\frac{2 l_{m} l_{s} k_{c} k_{s}}{J_{r} s^{2}-j H s-2 k_{h} l_{m}^{2}}, \\
G(s)=g_{a}(s) g_{f}(s)\left[g_{d}(s)+j 2 g_{\mathrm{cr}}(s)\right] e^{-\tau s} .
\end{gathered}
$$

Accordingly, the system open-loop translation function can be described as

$$
G(s) H(s)=\frac{2 l_{m} l_{s} k_{c} k_{s} g_{a}(s) g_{f}(s)\left[g_{d}(s)+j 2 g_{\mathrm{cr}}(s)\right] e^{-\tau s}}{J_{r} s^{2}-j H s-2 k_{h} l_{m}^{2}} .
$$

From (7), it can be found that, as for the high-frequency phase lag, there are four phase lag units in the whole open translation function, including switching power amplifier $g_{a}(s)$, antialias filter $g_{f}(s)$, digital control delay $e^{-\tau s}$, and the controlled objective delay. As for the phase lag at the low frequencies, the integral operation of the decentralized PID controller is the main factor.

According to the gyroscopic theory, the nutation frequency increases with the rotor speed. Contrarily, the precession frequency decreases with the rotor speed. Therefore, the high frequency phase lag is the key factor of the nutation instability of the MSR, and the low frequency phase lag is the cause of the precession instability.

Notice that the phase lag in low frequencies is limited and the low-pass filter of the filtered cross feedback control can effectively realize phase compensation; therefore, the precession stability of the MSR is relatively easy to be guaranteed. Contrarily, as for the phase lag at high frequencies, the larger the frequency is, the larger the phase lag is. Simultaneously, as for the same frequency, the larger the digital control delay is, the larger the phase lag is. Accordingly, compared with the precession instability, the nutation instability is a more challenging issue. Therefore, it is very necessary to develop high frequency phase compensation of the MSR.

\section{Modified Cross Feedback Control}

3.1. Strategy of the Proposed Controller. As for the MSR system with high speed, there are four possible phase compensation 
strategies according to (7). The first one is to compensate for the whole decentralized PID control channel. The second one is to compensate for the derivative item of the PID controller. The third one is to compensate for both the decentralized control channels and the cross feedback channels, which is the traditional compensation strategy. All of the three methods need four phase compensation filters since there are four coupling channels. The last one is to compensate for the cross feedback channels only, which needs only two phase compensation filters.

Define $\varphi(\omega)$ as the phase synthesis of the whole control channel; that is, $\varphi(\omega)=\angle G(j \omega)$, and $\theta(\omega)$ is the lag phase of the control channel without the controller; namely, $\theta(\omega)=$ $-\left(\angle g_{a}(j \omega)+\angle g_{f}(j \omega)+\angle e^{-\tau j \omega}\right)$. Therefore, $\theta(\omega)$ increases with $\omega$. As for the existing decentralized PID plus cross feedback control, note that the decentralized control channel and the cross feedback channel are of parallel connection; therefore, their phase lags are the same. For ease of presentation, $\theta_{1}(\omega)$ and $\theta_{2}(\omega)$ are employed to describe them, respectively; then we have $\theta_{1}(\omega)=\theta_{2}(\omega)=\theta(\omega)$.

According to the nutation stability criterion of the MSR [16], the sufficient and necessary condition of the nutation stability is that the phase of the control channel is within the range of 0 to 180 degrees. Note that the maximum leading phases of the cross feedback controller, $j 2 g_{\mathrm{cr}}(s)$, and the basic PID controller, $g_{d}(s)$, are within the range of 90 to 180 degrees and 0 to 90 degrees, respectively. Therefore when the phase synthesis of the whole control channel, $G(s)$, is zero degree, $\pi / 2<\theta(\omega)<\pi$. According to the principle of the cross feedback control, the cross feedback control is designed to counteract the gyroscopic effects; therefore, the effect of the cross item can be equivalent to $H \omega e^{j\left(180^{\circ}-\theta(\omega)\right)}$ theoretically.

Accordingly, under the condition $\pi / 2<\theta(\omega)<\pi$, the phase synthesis of the decentralized PID plus cross feedback controller can be described as

$$
\begin{array}{r}
\varphi(\omega) \\
=\arctan \left(\left(K_{d} \omega \cos \theta_{1}(\omega)-K_{p} \sin \theta_{1}(\omega)\right.\right. \\
\left.-\frac{K_{i}}{\omega} \cos \theta_{1}(\omega)+H \omega \sin \theta_{2}(\omega)\right) \\
\times\left(K_{p} \cos \theta_{1}(\omega)+K_{d} \omega \sin \theta_{1}(\omega)\right. \\
\left.\left.\quad-\frac{K_{i}}{\omega} \sin \theta_{1}(\omega)-H \omega \cos \theta_{2}(\omega)\right)^{-1}\right),
\end{array}
$$

where $K_{p}=2 l_{m} l_{s} k_{c} k_{s} k_{p}, K_{i}=2 l_{m} l_{s} k_{c} k_{s} k_{i}$, and $K_{d}=$ $2 l_{m} l_{s} k_{c} k_{s} k_{d}$, where $k_{p}, k_{i}$, and $k_{d}$ are the proportional, integral, and differential coefficients of the decentralized PID controller.

Note that the critical nutation frequency is high frequency; the phase effect of the integral is small enough to be ignored compared with that of the proportional and differential as well as the cross feedback control. Accordingly, (8) can be simplified as

$\varphi(\omega)$

$$
\approx \arctan \frac{K_{d} \omega \cos \theta_{1}(\omega)-K_{p} \sin \theta_{1}(\omega)+H \omega \sin \theta_{2}(\omega)}{K_{p} \cos \theta_{1}(\omega)+K_{d} \omega \sin \theta_{1}(\omega)-H \omega \cos \theta_{2}(\omega)} .
$$

Then

$$
\begin{gathered}
\frac{\partial \varphi(\omega)}{\partial \theta_{2}(\omega)} \approx \frac{1}{1+\xi^{2}} \frac{H \omega \cos \theta_{2}(\omega) \gamma-H \omega \sin \theta_{2}(\omega) \kappa}{\gamma^{2}}, \\
\frac{\partial \varphi(\omega)}{\partial \theta_{1}(\omega)} \approx \frac{1}{1+\xi^{2}}\left(\left(\left(-K_{d} \omega \sin \theta_{1}(\omega)-K_{p} \cos \theta_{1}(\omega)\right) \gamma\right.\right. \\
\left.-\left(-K_{p} \sin \theta_{1}(\omega)+K_{d} \omega \cos \theta_{1}(\omega)\right) \kappa\right) \\
\left.\times\left(\gamma^{2}\right)^{-1}\right),
\end{gathered}
$$

where

$$
\begin{aligned}
& \gamma=K_{p} \cos \theta_{1}(\omega)+K_{d} \omega \sin \theta_{1}(\omega)-H \omega \cos \theta_{2}(\omega), \\
& \kappa=K_{d} \omega \cos \theta_{1}(\omega)-K_{p} \sin \theta_{1}(\omega)+H \omega \sin \theta_{2}(\omega), \\
& \xi=\frac{K_{d} \omega \cos \theta_{1}(\omega)-K_{p} \sin \theta_{1}(\omega)+H \omega \sin \theta_{2}(\omega)}{K_{p} \cos \theta_{1}(\omega)+K_{d} \omega \sin \theta_{1}(\omega)-H \omega \cos \theta_{2}(\omega)} .
\end{aligned}
$$

Therefore, $\lim _{\varphi \rightarrow 0^{-}}(\kappa / \gamma)=0$.

Accordingly,

$$
\lim _{\varphi(\omega) \rightarrow 0^{-}} \frac{\partial \varphi(\omega) / \partial \theta_{2}(\omega)}{\partial \varphi(\omega) / \partial \theta_{1}(\omega)} \approx \frac{H \omega \cos \theta_{2}(\omega)}{-K_{d} \omega \sin \theta_{1}(\omega)-K_{p} \cos \theta_{1}(\omega)}
$$

$$
\text { Let } \varphi(\omega)=0 \text {; we have }
$$

$$
K_{d} \omega \cos \theta_{1}(\omega)-K_{p} \sin \theta_{1}(\omega)+H \omega \sin \theta_{1}(\omega)=0
$$

Note that, under the condition $\pi / 2<\theta(\omega)<\pi$, $\cos \theta(\omega) \neq 0$ yields

$$
\tan \theta(\omega)=\frac{K_{d} \omega}{K_{p}-H \omega}
$$

Substituting (14) into (12) produces

$$
\begin{aligned}
\lim _{\varphi(\omega) \rightarrow 0^{-}} \frac{\partial \varphi(\omega) / \partial \theta_{2}(\omega)}{\partial \varphi(\omega) / \partial \theta_{1}(\omega)} & \approx \frac{H \omega}{K_{d}^{2} \omega^{2}\left(1 /\left(H \omega-K_{p}\right)\right)-K_{p}} \\
& =\frac{H^{2} \omega^{2}-H \omega K_{p}}{K_{d}^{2} \omega^{2}-K_{p}^{2}-H \omega K_{p}} .
\end{aligned}
$$




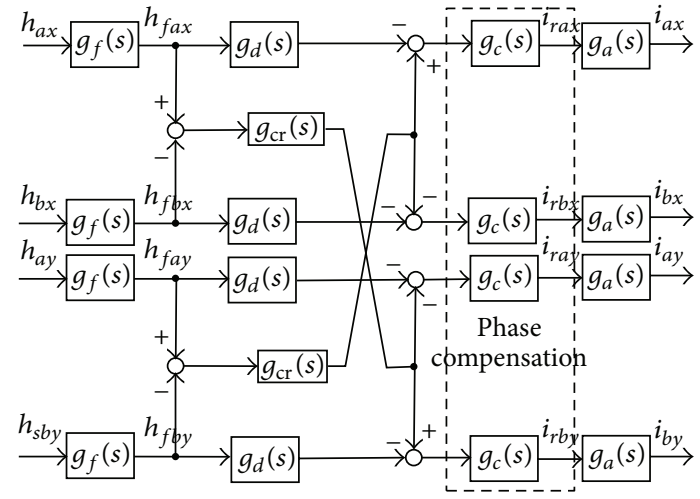

FIGURE 3: Schematic diagram of the cross feedback controller with phase compensation of coupling channels.

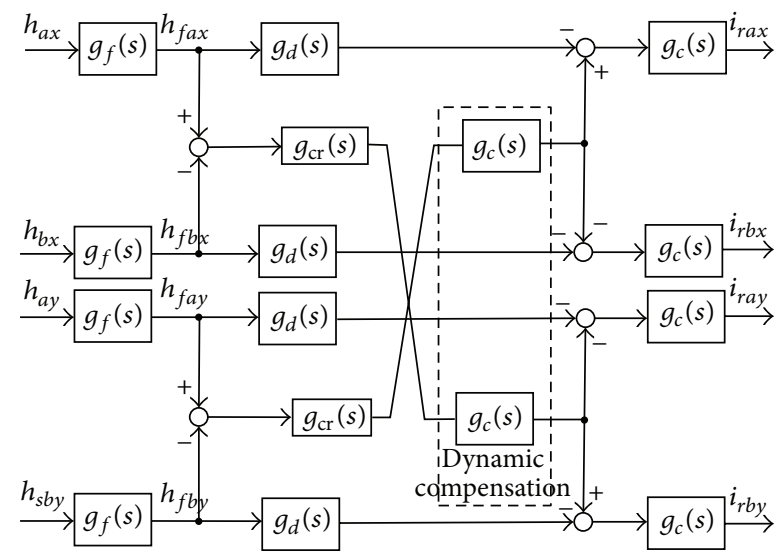

FIGURE 4: Schematic diagram of the modified cross feedback control based on phase compensation of cross channels.

Note that $H \omega \gg K_{p}$ and $H^{2} \omega^{2} \gg K_{d}^{2} \omega^{2}-K_{p}^{2}$ at high rotating speeds, $\left(H^{2} \omega^{2}-H \omega K_{p}\right) /\left(K_{d}^{2} \omega^{2}-K_{p}^{2}-H \omega K_{p}\right) \gg 1$. Therefore, as for the MSR with high speed, compared with the phase lag of the decentralized channel, the phase lag of the cross channels is the main factor which influences the whole decentralized PID plus cross feedback controller.

According to the analysis above, we can draw a conclusion that, compared with the other compensation methods, the phase compensation based on cross feedback channel can give attention to both the improvement of nutation stability and rejection of noise amplification. Just based on this, a modified cross feedback controller with phase compensation of cross channel is presented in this paper.

\subsection{Modified Cross Feedback Controller with Phase Compen-} sation of Cross Channel. Figures 3 and 4 show the traditional phase compensation strategy based on coupling channels and the proposed modified cross feedback control strategy based on cross channel, respectively. Compared with the traditional cross feedback control (see Figure 1), the dynamic compensation filter $g_{c}(s)$ is introduced into the cross channel. Different from the existing cross feedback control with phase compensation of cross channel, the presented method does not need to compensate for every radial channel of the MSR. Only the two rotation modes of the MSR need to be compensated instead of the four modes (two rotation modes and two translation modes). That is, there are only two compensation filters instead of four. Therefore, compared to the traditional methods, computation resources and noise amplification introduced by the phase compensation can be greatly reduced.

Combing (6), the control channel transfer function after dynamic compensation can be described as

$$
G_{c}(s)=g_{a}(s) g_{f}(s)\left[g_{d}(s)+j 2 g_{\mathrm{cr}}(s) g_{c}(s)\right] e^{-\tau s} .
$$

In is necessary to note that the quantitative analysis of the phase compensation filter $g_{c}(s)$ can be further developed by the nutation stability criterion of MSR [16] in practice.

As for the laboratory setup (Figure 6), its rated rotor speed is $200 \mathrm{~Hz}$, and its nutation frequency needed to be controlled is about $290 \mathrm{~Hz}$. The frequency responses of the whole control channel can be measured via a sine sweep test using a dynamic signal analyzer. Then we can get the phase lag at the rated nutation frequency. According to system nutation stability criterion [16] and the desired performances, we can easily resolve the least phase needed to be compensated. Based on these, considering the simplicity of realization and noise rejection, a second-order filter can be designed, whose transfer function is written as

$$
g_{c}(s)=\frac{5.3 s^{2}+8600 s+1.2 \times 10^{7}}{2.5 s^{2}+5200 s+1.2 \times 10^{7}}
$$

According to the nutation stability criterion of the MSR [16], the positive-frequency response curve can reveal the system nutation stability, including the critical nutation frequency and its corresponding critical rotor speed. The frequency response comparisons among the three methods are shown in Figure 5, which is drawn with MATLAB. From Figure 5, compared with the cross feedback control without phase compensation, the magnitude and the phase lag of the control channel around $290 \mathrm{~Hz}$ have been compensated effectively. According to the stability criterion of the nutation mode, the critical nutation frequency has been increased from $300 \mathrm{~Hz}$ to $468 \mathrm{~Hz}$, which means that the critical rotor speed of nutation mode can be greatly improved. Although the phase compensation method of radial channels can also increase the critical nutation frequency to about $468 \mathrm{~Hz}$, its corresponding amplitude of the amplitude response curve at this frequency is larger than that of the proposed one. Also according to the critical rotor speed of the nutation mode [16], the critical rotor speed of the nutation mode is smaller than that of the proposed method. Additionally, from Figure 5, it can be seen that the amplitude of the radial channel compensation method is far larger than that of the proposed one in the whole high frequency range, which inevitably introduces extra system noise.

Note that this phase compensation strategy adapts to not only the decentralized PID plus filtered cross feedback control, but also other kinds of cross decoupling control method, such as speed cross, filtered cross feedback control. 


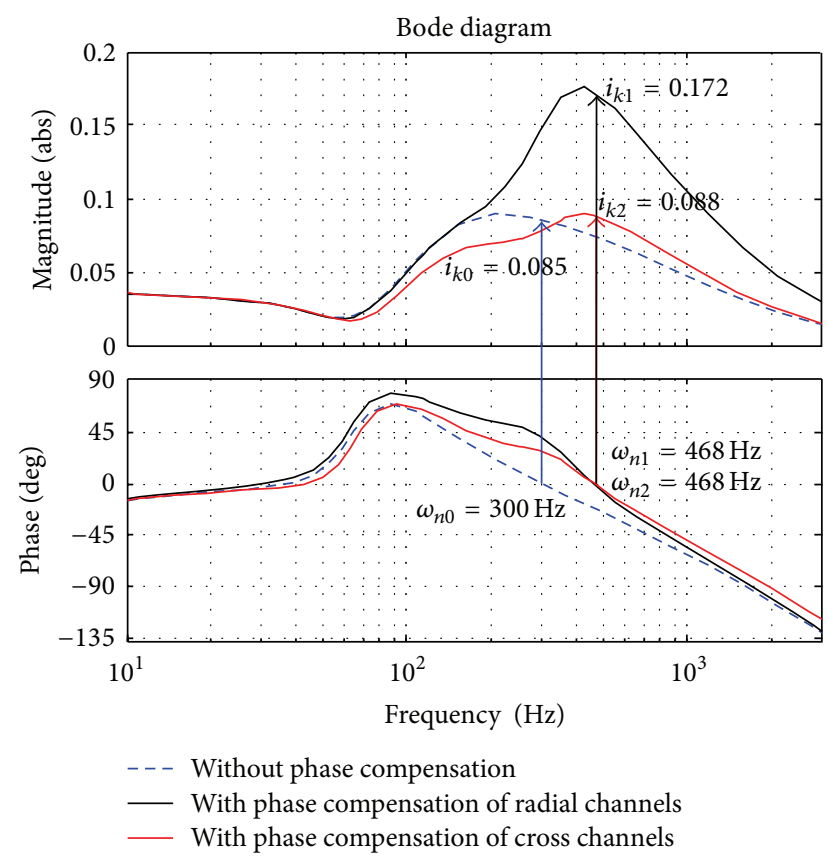

Figure 5: Frequency response comparison of control channel.

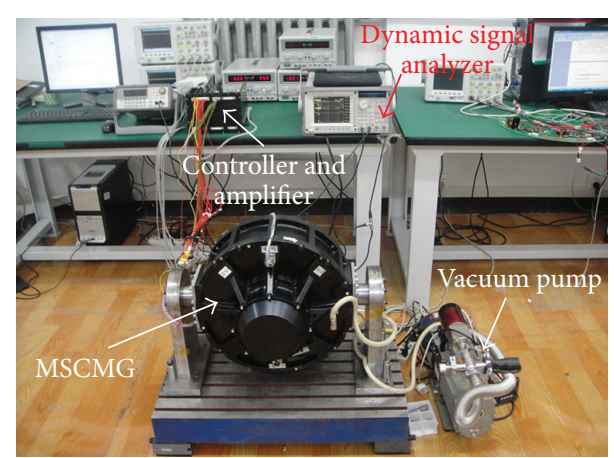

FIGURE 6: Photograph of the fabricated laboratory setup.

3.3. Robustness to Model Errors of the Proposed Method. As for the linear stationary system of the rotational modes of the MSR, it can be described in the following differential operator equations:

$$
\begin{gathered}
\mathbf{T}(D) \mathbf{x}=\mathbf{U}(D) \mathbf{u}, \\
\mathbf{y}=\mathbf{V}(D) \mathbf{x}+\mathbf{W}(D) \mathbf{u},
\end{gathered}
$$

where, $\mathbf{u}$ is 2 -dimensional-input vector $\left[\begin{array}{ll}i_{\beta} & i_{\alpha}\end{array}\right]^{\mathrm{T}}, \mathbf{y}$ is 2 dimensional-output vector $[\beta \alpha]^{\mathrm{T}}, \mathbf{x}$ is the state vector of the system $\left[\begin{array}{ll}\beta & \alpha\end{array}\right]^{\mathrm{T}}, D$ is the differential operator, and $\mathrm{T}(D)$, $\mathbf{U}(D), \mathbf{W}(D)$, and $\mathbf{V}(D)$ are, respectively, the polynomial of $D$. The Laplace transformation of (18) under the zero initial condition is

$$
\begin{gathered}
\mathbf{T}(s) \mathbf{x}=\mathbf{U}(s) \mathbf{u}, \\
\mathbf{y}=\mathbf{V}(s) \mathbf{x}+\mathbf{W}(s) \mathbf{u},
\end{gathered}
$$

where

$$
\begin{gathered}
\mathbf{T}(s)=\left[\begin{array}{cc}
J_{r} s^{2}-2 k_{h} l_{m}^{2} & -J_{z} \Omega s \\
J_{z} \Omega s & J_{r} s^{2}-2 k_{h} l_{m}^{2}
\end{array}\right], \\
\mathbf{U}(s)=\left[\begin{array}{cc}
2 l_{m} l_{s} k_{i} k_{s} & 0 \\
0 & 2 l_{m} l_{s} k_{i} k_{s}
\end{array}\right], \\
\mathbf{W}(s)=\mathbf{0}^{2 \times 2}, \quad \mathbf{V}(s)=\mathbf{I}^{2 \times 2} .
\end{gathered}
$$

Accordingly, the transfer function matrix of the controlled plant can be resolved as

$$
\begin{aligned}
\mathbf{P}(s)= & \mathbf{V}(s) \mathbf{T}^{-1}(s) \mathbf{U}(s)+\mathbf{W}(s) \\
= & \frac{2 l_{m} l_{s} k_{i} k_{s}}{\left(J_{r} s^{2}-2 k_{h} l_{m}^{2}\right)^{2}+\left(J_{z} \Omega s\right)^{2}} \\
& \times\left[\begin{array}{cc}
J_{r} s^{2}-2 k_{h} l_{m}^{2} & J_{z} \Omega s \\
-J_{z} \Omega s & J_{r} s^{2}-2 k_{h} l_{m}^{2}
\end{array}\right] .
\end{aligned}
$$

According to Figure 4, the transfer function matrix of the forward channel of the control system can be written as

$$
\mathbf{K}(s)=g_{a}(s) e^{-\tau s}\left[\begin{array}{cc}
g_{d}(s) & g_{\mathrm{cr}}(s)+g_{\mathrm{com}}(s) \\
-g_{\mathrm{cr}}(s)-g_{\mathrm{com}}(s) & g_{d}(s)
\end{array}\right] .
$$

Therefore the open-loop transfer function matrix of the MIMO system can be resolved as

$$
\begin{aligned}
\mathbf{Q}(s) & =\mathbf{g}_{f}(\mathbf{s}) \mathbf{P}(s) \mathbf{K}(s) \\
& =\frac{2 l_{m} l_{s} k_{i} k_{s} g_{a}(s) g_{f}(s) e^{-\tau s}}{\left(J_{r} s^{2}-2 k_{h} l_{m}^{2}\right)^{2}+\left(J_{z} \Omega s\right)^{2}}\left[\begin{array}{cc}
\sigma_{1}(s) & \sigma_{2}(s) \\
-\sigma_{2}(s) & \sigma_{1}(s)
\end{array}\right],
\end{aligned}
$$

where

$$
\begin{aligned}
& \sigma_{1}(s)=\left(J_{r} s^{2}-2 k_{h} l_{m}^{2}\right) g_{d}(s)-J_{z} \Omega s\left(g_{\mathrm{cr}}(s)+g_{\mathrm{com}}(s)\right), \\
& \sigma_{2}(s)=\left(J_{r} s^{2}-2 k_{h} l_{m}^{2}\right)\left(g_{\mathrm{cr}}(s)+g_{\mathrm{com}}(s)\right)+J_{z} \Omega g_{d}(s) s .
\end{aligned}
$$

Accordingly, the open-loop transfer function matrix of the MIMO system is a normal algebra matrix [14]. According to the multiple-variable frequency-domain theory [18], the system has good robust performance to model errors since the open-loop transfer function matrix is a normal matrix. Therefore, the proposed phase compensation method is robust to model errors of the controlled plant.

\section{Simulation and Experimental Results}

In order to demonstrate the effectiveness and validity of the proposed control strategy and to reveal how closely the theory represented the physical system, both simulation and experiments have been developed. 
4.1. Simulation and Experimental Setup. As for the hardware realization is concerned, a TMS320C31 digital signal processor (DSP) is employed. Both the sampling time and servo time are set to $150 \mu \mathrm{s}$ and the switching period is set to $50 \mu \mathrm{s}$. The controller is well integrated in power electronics, which operates with a supply voltage of $28 \mathrm{~V} \mathrm{DC}$.

Considering the interest bandwidths of the current loop and the position loop of the MSR and the simplicity of the realization, second-order antialias filters are introduced into both the displacement and current AD sampling of the MSR system, whose transfer function is given as

$$
g_{f}(s)=\frac{1}{\left(3.3 \times 10^{-5} s+1\right)^{2}}
$$

For fair comparison of the decoupling, stability, and computer run time of the different methods, both the proposed controller and the channel decoupling approach employ the same decentralized PID plus cross feedback controller and the same phase compensation filter described as (17). The main system parameters and the controller coefficients used in simulations and experiments are listed in Tables 1 and 2 in the Appendix.

The computer run time, that is, the computation delay, of the two methods is compared, and the test results are given in Table 3. From Table 3, the proposed method has a much shorter computer run time than that of the radial channel compensation one. This verifies that the modified cross feedback control method can largely simplify the industrial realization with smaller computer resources than that of the existing radial channel compensation, method, which is in accordance with the analysis in Section 1.

4.2. Stability Performance and Noise Level. To verify the effectiveness of the proposed control strategy to improve the system stability, rotor speed root locus method is employed. As for the rotor speed root locus, the speed range is from 0 to $350 \mathrm{~Hz}$, and it is drawn every $7 \mathrm{~Hz}$. Figure 7 gives the comparative results among the cross feedback control without phase compensation, the cross feedback control with the radial channel phase compensation and the modified cross feedback control strategy.

From Figure 7, as for the controller without phase compensation, the critical rotor speed of the nutation stability is only $203 \mathrm{~Hz}$ and the critical rotor speed of precession mode can reach to $315 \mathrm{~Hz}$. Obviously, the nutation stability is the main contradictory for the traditional cross feedback controller, which is in agreement with the analysis in Section 1. From Figure 7(b), after employing the radial channel phase compensation, the critical rotor speeds of the nutation and precession modes can increase to about $315 \mathrm{~Hz}$ and $322 \mathrm{~Hz}$, respectively. With the proposed method, the critical nutation rotor speed can be further increased to about $336 \mathrm{~Hz}$, and the critical precession rotor speed has remained approximately constant. This indicates that the proposed method can greatly improve the stability of the nutation mode, improving effectively the system stability.
TABLE 1: System parameters of the MSCMG.

\begin{tabular}{lc}
\hline Parameter & Value \\
\hline$m$ & $56 \mathrm{~kg}$ \\
$J_{y}$ & $0.6032 \mathrm{~kg} \cdot \mathrm{m}^{2}$ \\
$l_{s}$ & $0.177 \mathrm{~m}$ \\
$k_{i 0}$ & $1140 \mathrm{~N} / \mathrm{A}$ \\
$J_{x}$ & $0.6032 \mathrm{~kg} \cdot \mathrm{m}^{2}$ \\
$J_{z}$ & $0.7958 \mathrm{~kg} \cdot \mathrm{m}^{2}$ \\
$l_{m}$ & $0.113 \mathrm{~m}$ \\
$k_{h 0}$ & $2.83 \mathrm{~N} / \mu \mathrm{m}$ \\
\hline
\end{tabular}

TABLE 2: Parameters of the controller.

\begin{tabular}{lc}
\hline Parameter & Value \\
\hline$k_{a m}$ & 2.0 \\
$k_{p}$ & 2.5 \\
$k_{d}$ & 0.008 \\
$k_{l c}$ & 0.00015 \\
$f_{h}$ & $300 \mathrm{~Hz}$ \\
$i_{c}$ & 10 \\
$k_{i}$ & 25 \\
$k_{h c}$ & 0.0045 \\
$k_{\mathrm{cr}}$ & 1.0 \\
$f_{l}$ & $30 \mathrm{~Hz}$ \\
\hline
\end{tabular}

TABLE 3: Computer run time of different control methods.

\begin{tabular}{lc}
\hline Method & Computer run time \\
\hline $\begin{array}{l}\text { Modified cross feedback control based on } \\
\text { phase compensation of cross channels }\end{array}$ & $108.1 \mu \mathrm{s}$ \\
$\begin{array}{l}\text { Cross feedback control based on phase } \\
\text { compensation of radial channels }\end{array}$ & $135.2 \mu \mathrm{s}$ \\
\hline
\end{tabular}

Figures 8 and 9 show the radical displacement responses of the MSR with the cross feedback control with the traditional phase compensation and the modified cross feedback control method, respectively. Comparing Figure 8(a) with Figure 9(a), it is obvious that the peak-peak value of the radial displacements has been greatly decreased. Also, comparing Figure 9(a) with Figure 9(b), the nutation amplitude of channel $A_{x}$ has been decreased from about $-40 \mathrm{~dB}$ to $-44 \mathrm{~dB}$, and the frequency spectrum noise has been greatly alleviated too by employing the proposed cross feedback control method. All of these demonstrate that the modified cross feedback control method cannot only improve the system nutation stability, but also can alleviate the system noises, which is in accordance with the analysis made above.

From Figures 8 and 9, it can be found that the peakpeak amplitude of the radial channel with the proposed method is only about $10 \mu \mathrm{m}$. Contrarily, it is about $15 \mu \mathrm{m}$ with the channel method. To be sure, it is dubious to judge the decoupling performance of the controller presented in this paper simply by the peak-peak amplitude since a good control performance may be achieved with the traditional controller by selecting carefully controller coefficients. However, 


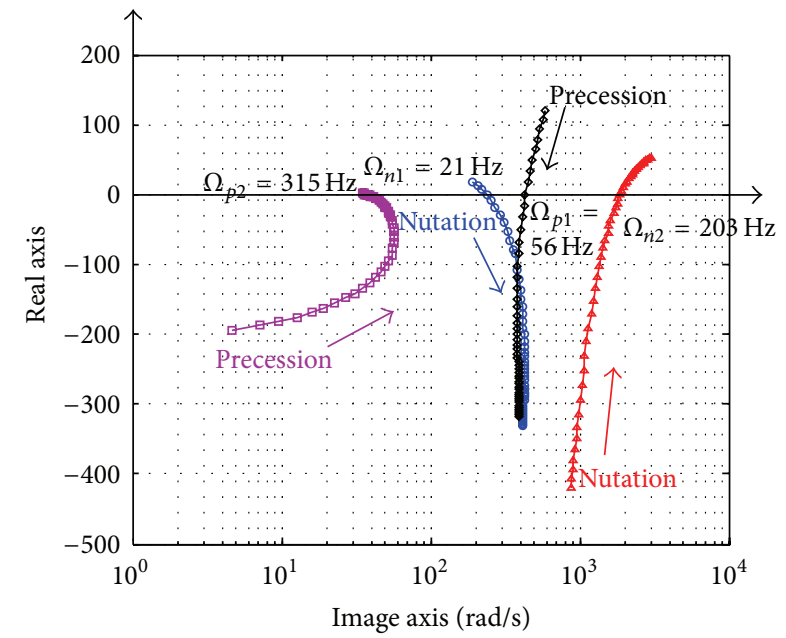

(a)

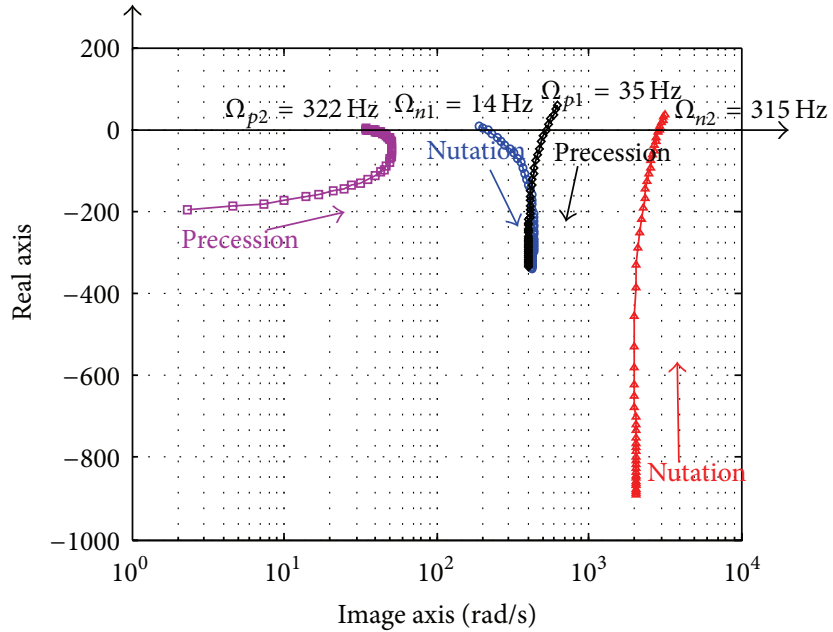

(b)

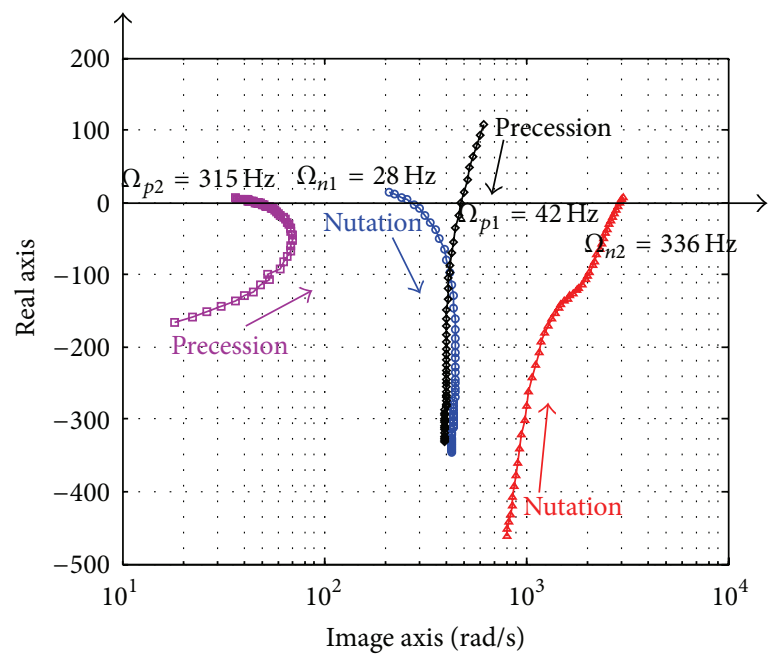

(c)

FIGURE 7: Rotor speed root locus comparison for the MSR with the increase of the rotor speed. (a) Cross feedback control without phase compensation. (b) Cross feedback control with traditional phase compensation method. (c) Modified cross feedback control. Note: the arrowhead denotes the increased direction of rotor speed.
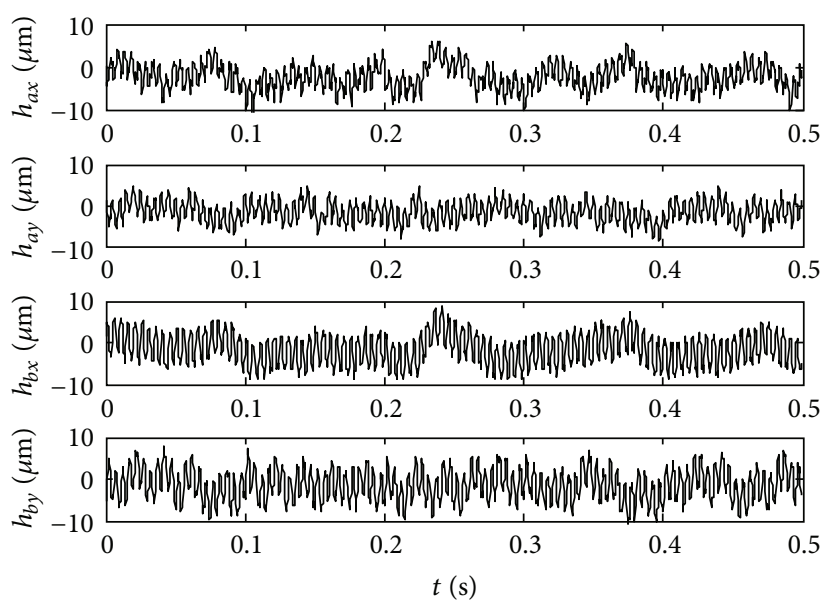

(a)

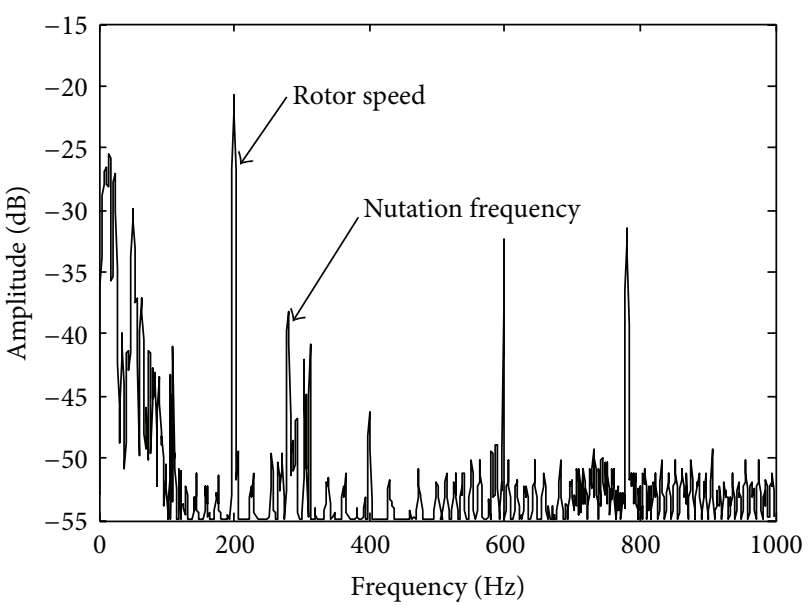

(b)

FIGURE 8: Experimental results of the cross feedback control with the traditional phase compensation method. (a) Radical displacement responses of the MSR. (b) Frequency spectrum of displacement $h_{a x}$. 


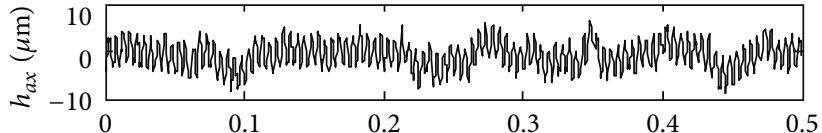

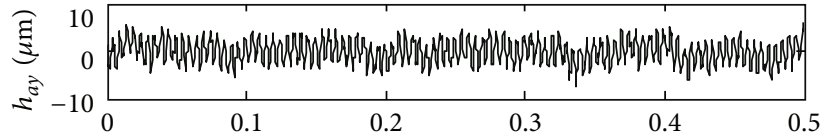
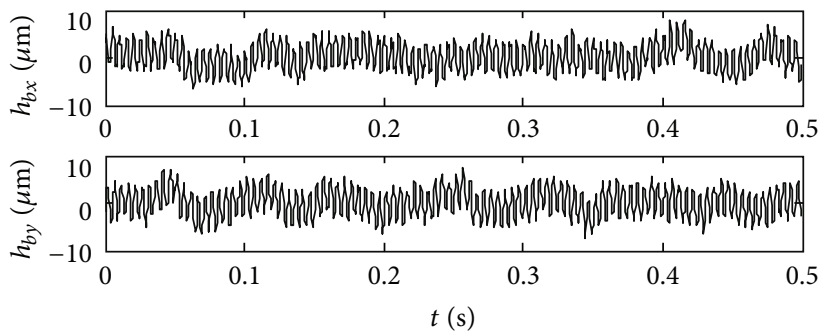

(a)

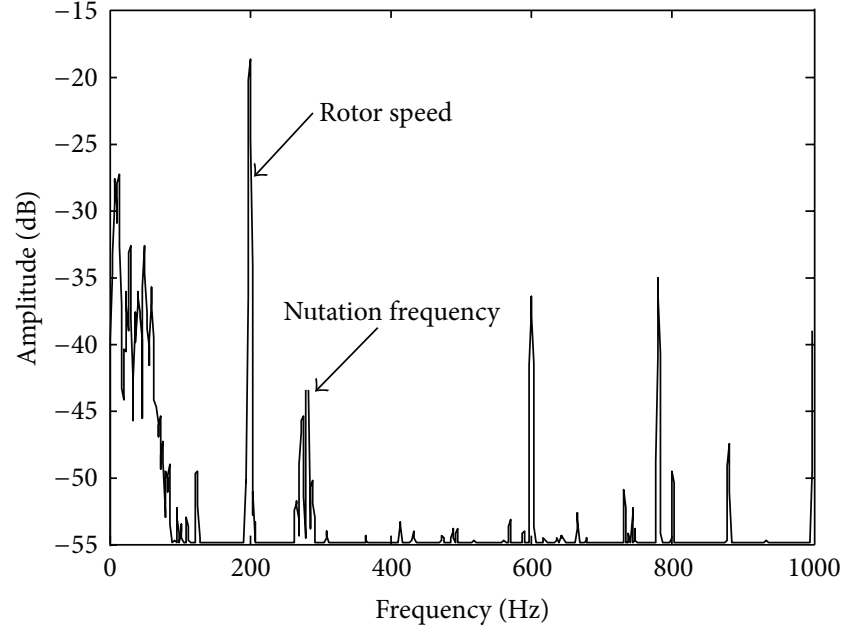

(b)

FIGURE 9: Experimental results of the modified cross feedback control method. (a) Radical displacement responses of the MSR. (b) Frequency spectrum of displacement $h_{a x}$.
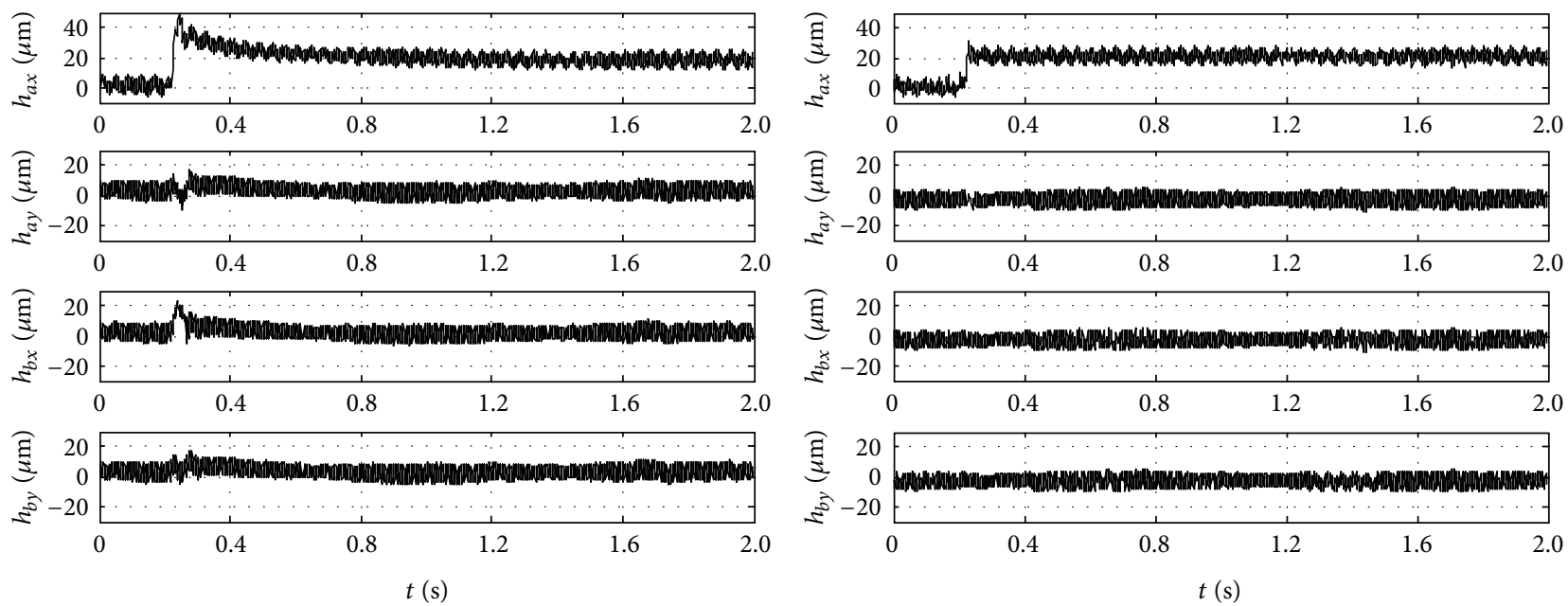

(a)

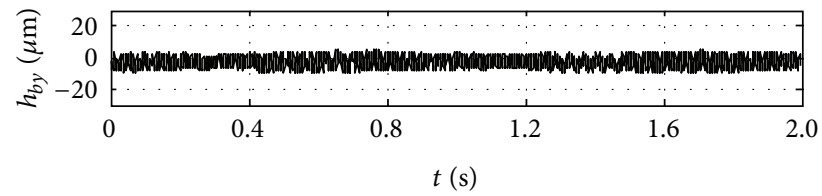

(b)

FIGURE 10: Experimental comparison of displacement responses based on two different controllers under the condition $\Omega=12000 \mathrm{r} / \mathrm{min}$.

(a) Decentralized PID plus cross decoupling control. (b) The proposed control.

the changes on the steady-state peak-peak amplitude with the same controller and the same coefficients can undoubtedly indicate the precision performance of a controller. In fact, for the traditional control, the steady-state precision is yet relatively high. Extra control system delay degrades the decoupling performance and extra high-pass filters result in extra system noise.

4.3. Decoupling Performance. In order to further prove that the proposed method can effectively improve the system decoupling performance, comparative simulations and experiments have been developed too.

Under the condition $\Omega=10000 \mathrm{r} / \mathrm{min}$, at time $t=0.2 \mathrm{~s}$, the reference displacement of channel $A_{x}$ varies from 0 to
$20 \mu \mathrm{m}$; the comparative experimental results are shown in Figure 10.

Obviously, the simulation and experimental results are in good agreement too. As seen in Figure 10(a), the step responses of channel $A_{x}$ with the traditional controller result in distinct fluctuations within the other three channels. On the other hand, the proposed controller brings few fluctuations into the other three channels, as shown in Figure 10(b). That is, one displacement step input almost does not affect the other three outputs. Therefore, it can be suggested that compared with the traditional cross feedback control the modified method can realize higher decoupling precision than that of the traditional method, which also agrees very well with the analysis in Section 2. 
In fact, the traditional decentralized PID plus cross decoupling control can restrain the coupling between $x$ - and $y$-directions to a certain degree. However, it cannot completely eliminate the coupling owing to the phase lag of the control system.

\section{Conclusion}

To improve the nutation stability and the decoupling performances of the high-speed MSR with significant gyroscopic effects, this paper proves at first that the phase lag of the cross channels is often the main factor influencing the system nutation stability. Then a modified cross feedback control based on the dynamic compensation of the cross feedback channel is proposed, and its robustness to model errors is analyzed. Simulation and experimental results demonstrate that compared with the traditional channel compensation method, the presented one not only can effectively enhance the stability abundance and the decoupling performances, but also can reduce computation resources and reject the system noises greatly.

\section{Appendix}

In Table $1, k_{i 0}$ and $k_{h 0}$ are the current-force factor and displacement-force factor of the radial magnetic bearings under the conditions $i_{\gamma}=0$ and $h_{m \gamma}=0(\gamma=a x, a y, b x, b y)$; $R$ and $L$ are the coil resistance and inductance of the radial magnetic bearings.

In Table 2, $k_{a m}$ and $i_{c}$ are the proportional and feedback coefficients of the current controller for the MSR system, which are the same as that of the proposed controller; $k_{h c}$ and $k_{l c}$ are the cross coefficients of the high-pass and lowpass filters, respectively; $k_{\mathrm{cr}}$ is the total cross coefficient; $f_{h}$ and $f_{l}$ are the cut-off frequencies of the two-order high-pass and low-pass filters.

\section{Conflict of Interests}

The authors declare that there is no conflict of interests regarding the publication of this paper.

\section{Acknowledgments}

This work was supported in part by the National Basic Research Program of China, under Grant 2009CB72400101C, and by the National Natural Science Foundation of China.

\section{References}

[1] S. P. Bhat and P. K. Tiwari, "Controllability of spacecraft attitude using control moment gyroscopes," IEEE Transactions on Automatic Control, vol. 54, no. 3, pp. 585-590, 2009.

[2] M. D. Carpenter and M. A. Peck, "Dynamics of a high-agility, low-power imaging payload," IEEE Transactions on Robotics, vol. 24, no. 3, pp. 666-675, 2008.

[3] B. Thornton, T. Ura, Y. Nose, and S. Turnock, "Zero-G class underwater robots: Unrestricted attitude control using control moment gyros," IEEE Journal of Oceanic Engineering, vol. 32, no. 3, pp. 565-583, 2007.

[4] M. D. Carpenter and M. A. Peck, "Reducing base reactions with gyroscopic actuation of space-robotic systems," IEEE Transactions on Robotics, vol. 25, no. 6, pp. 1262-1270, 2009.

[5] T. Schuhmann, W. Hofmann, and R. Werner, "Improving operational performance of active magnetic bearings using Kalman filter and state feedback control," IEEE Transactions on Industrial Electronics, vol. 59, no. 2, pp. 821-829, 2012.

[6] J. Fang and Y. Ren, "High-precision control for a single-gimbal magnetically suspended control moment gyro based on inverse system method," IEEE Transactions on Industrial Electronics, vol. 58, no. 9, pp. 4331-4342, 2011.

[7] S.-L. Chen and C.-C. Weng, "Robust control of a voltagecontrolled three-pole active magnetic bearing system," IEEE/ASME Transactions on Mechatronics, vol. 15, no. 3, pp. 381-388, 2010.

[8] J. Fang and Y. Ren, "Decoupling control of magnetically suspended rotor system in control moment gyros based on an inverse system method," IEEE/ASME Transactions on Mechatronics, vol. 17, pp. 1133-1144, 2012.

[9] J. Fang and Y. Ren, "Self-adaptive phase-lead compensation based on unsymmetrical current sampling resistance network for magnetic bearing switching power amplifiers," IEEE Transactions on Industrial Electronics, vol. 59, no. 2, pp. 1218-1227, 2012.

[10] J.-W. Kim, S.-Y. Yoo, Y.-C. Bae, and M. D. Noh, "Optimal design of magnetically-levitated flywheel energy storage system considering system stability," in Proceedings of the ICROS-SICE International Joint Conference (ICCAS-SICE '09), pp. 44014406, Fukuoka, Japan, August 2009.

[11] M. Ahrens, L. Kučera, and R. Larsonneur, "Performance of a magnetically suspended flywheel energy storage device," IEEE Transactions on Control Systems Technology, vol. 4, no. 5, pp. 494-502, 1996.

[12] G. V. Brown, A. Kascak, R. H. Jansen, T. P. Dever, and K. P. Duffy, "Stabilizing gyroscopic modes in magnetic-bearing-supported flywheels by using cross-axis proportional gains," in Proceedings of the AIAA Guidance, Navigation, and Control Conference, pp. 1132-1143, AIAA, San Francisco, Calif, USA, August 2005.

[13] T. P. Dever, G. V. Brown, K. P. Duffy, and R. H. Jansen, "Modeling and development of a magnetic bearing controller for a high speed flywheel system," in Proceedings of the 2nd International Energy Conversion Engineering Conference, pp. 888-899, AIAA, Providence, RI, USA, August 2004.

[14] Y. Ren, D. Su, and J. Fang, "Whirling modes stability criterion for a magnetically suspended flywheel rotor with significant gyroscopic effects and bending modes," IEEE Transactions on Power Electronics, vol. 28, pp. 5890-5901, 2013.

[15] Y. Ren and J. Fang, "Current-sensing resistor design to include current derivative in PWM H-bridge unipolar switching power amplifiers for magnetic bearings," IEEE Transactions on Industrial Electronics, vol. 59, pp. 4590-4600, 2012.

[16] J. Fang, Y. Ren, and Y. Fan, "Nutation and precession stability criterion of magnetically suspended rigid rotors with gyroscopic effects based on positive and negative frequency characteristics," IEEE Transactions on Industrial Electronics, vol. 61, pp. 2003-2014, 2014. 
[17] Y. Ren and J. Fang, "High-precision and strong-robustness control for a MSCMG based on modal separation and rotation motion decoupling strategy," IEEE Transactions on Industrial Electronics, vol. 61, pp. 1539-1551, 2014.

[18] A. Postlethwaite and G. J. MacFarlane, A Complex Variable Approach To the Analysis of Linear Multivariable Feedback Systems, Springer, Berlin, Germany, 1979. 


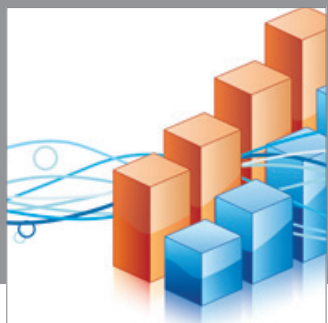

Advances in

Operations Research

mansans

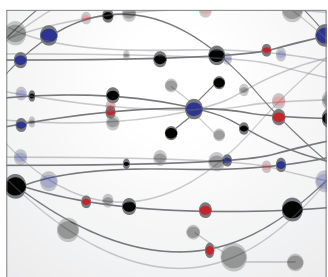

The Scientific World Journal
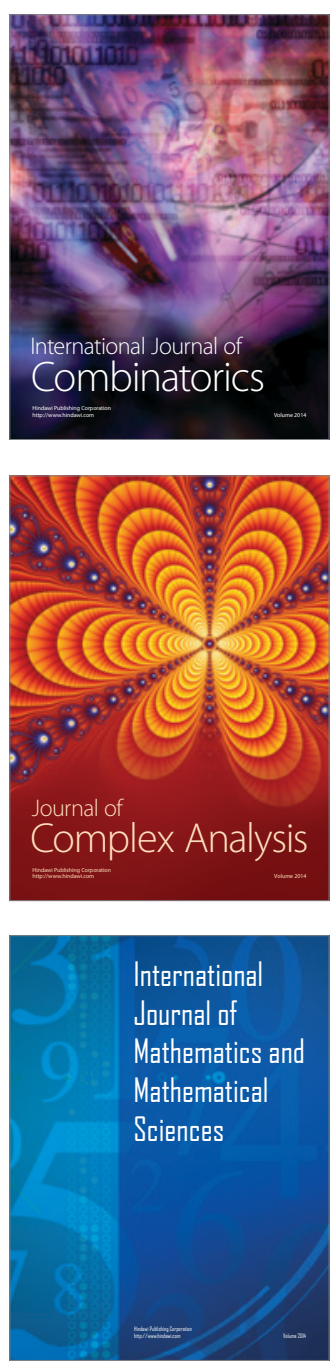
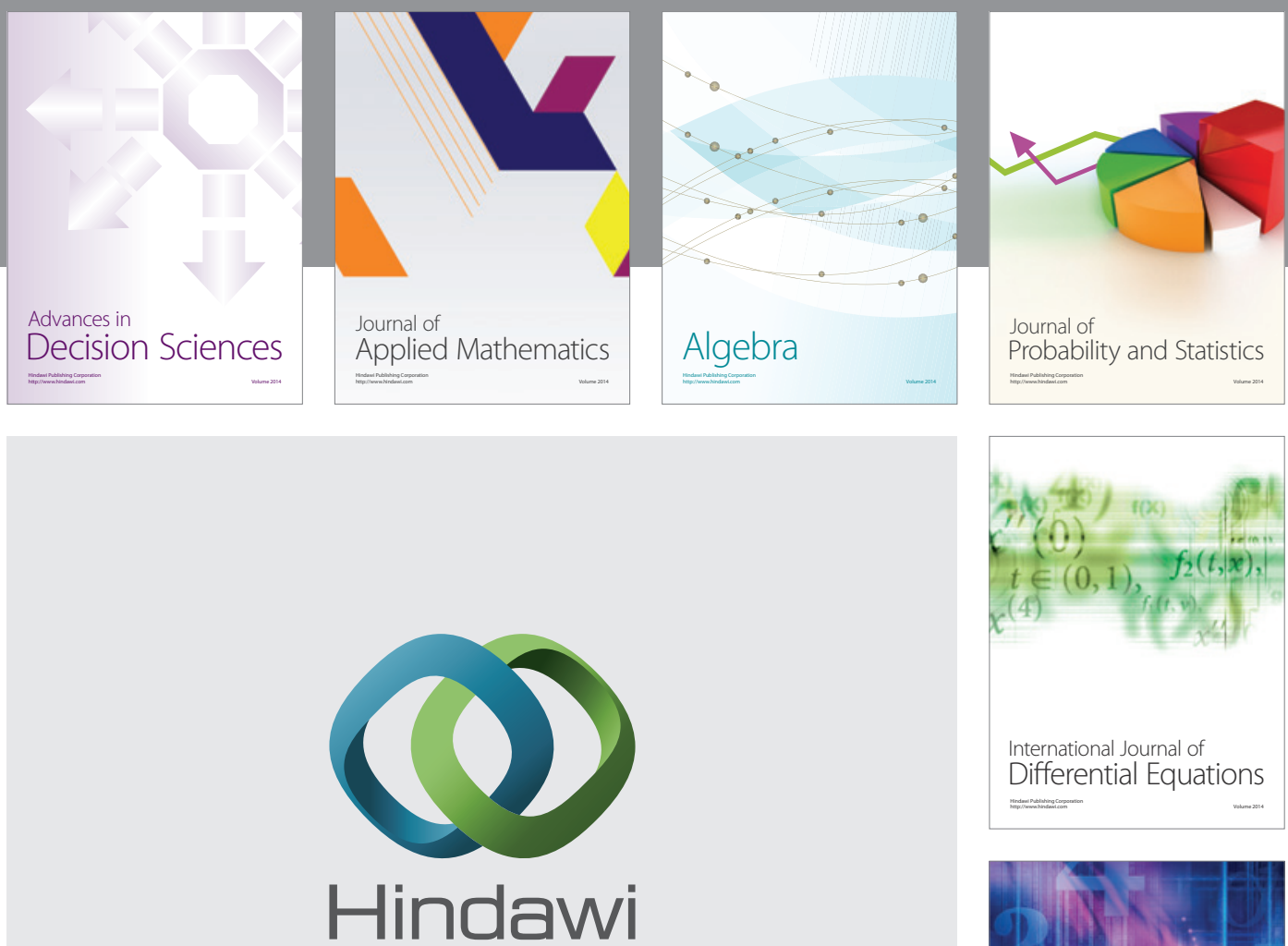

Submit your manuscripts at http://www.hindawi.com
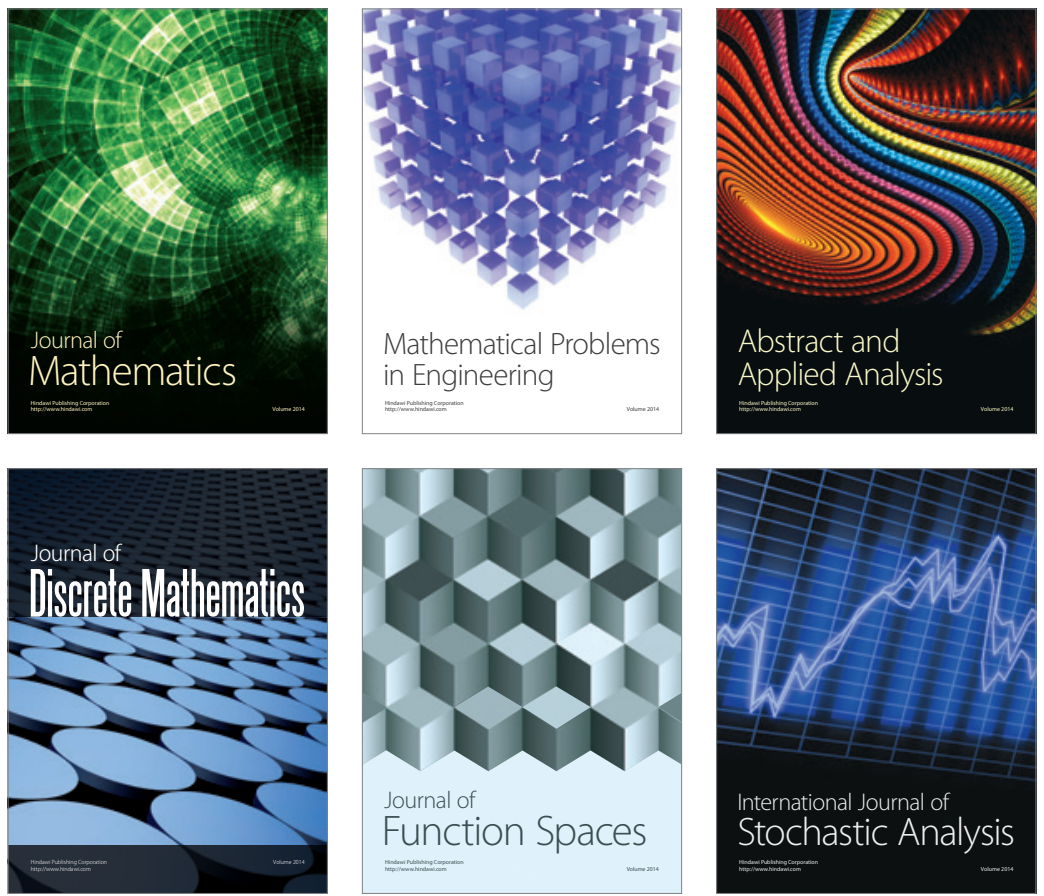

Journal of

Function Spaces

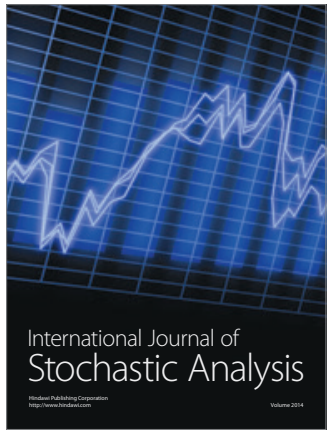

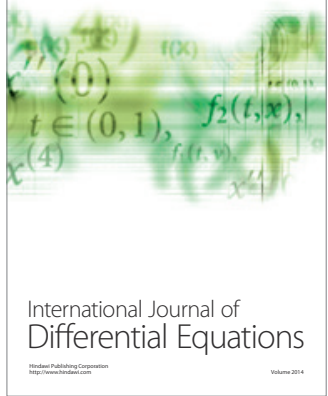
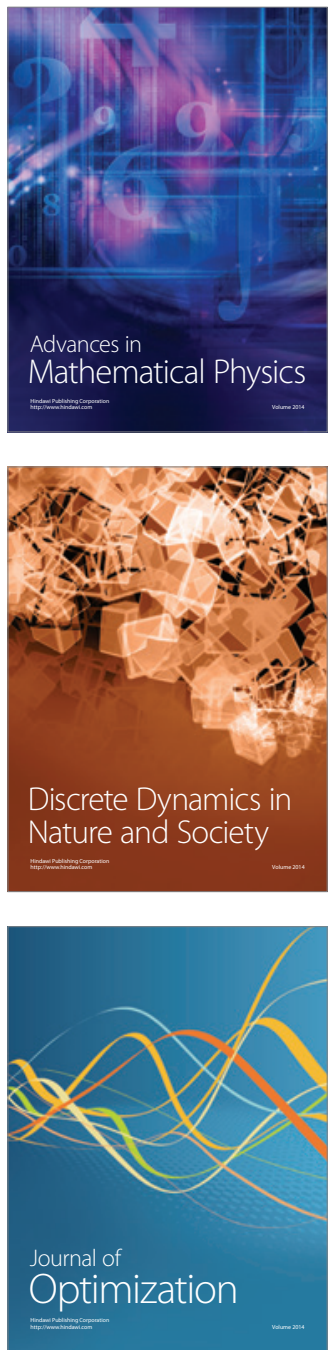